\title{
On the connection between dissipation enhancement in the ocean surface layer and Langmuir circulations
}

Article

Published Version

Teixeira, M. A. C. (2011) On the connection between dissipation enhancement in the ocean surface layer and Langmuir circulations. Journal of Physical Oceanography, 41 (10). pp. 2000-2007. ISSN 0022-3670 doi:

https://doi.org/10.1175/JPO-D-11-062.1 Available at https://centaur.reading.ac.uk/29238/

It is advisable to refer to the publisher's version if you intend to cite from the work. See Guidance on citing.

Published version at: http://dx.doi.org/10.1175/JPO-D-11-062.1

To link to this article DOI: http://dx.doi.org/10.1175/JPO-D-11-062.1

Publisher: American Meteorological Society

All outputs in CentAUR are protected by Intellectual Property Rights law, including copyright law. Copyright and IPR is retained by the creators or other copyright holders. Terms and conditions for use of this material are defined in the End User Agreement.

www.reading.ac.uk/centaur 
Central Archive at the University of Reading

Reading's research outputs online 


\title{
On the Connection between Dissipation Enhancement in the Ocean Surface Layer and Langmuir Circulations
}

\author{
Miguel A. C. TEIXEIRA \\ CGUL, IDL, University of Lisbon, Lisbon, Portugal
}

(Manuscript received 23 March 2011, in final form 21 June 2011)

\begin{abstract}
A mechanism for the enhancement of the viscous dissipation rate of turbulent kinetic energy (TKE) in the oceanic boundary layer (OBL) is proposed, based on insights gained from rapid-distortion theory (RDT). In this mechanism, which complements mechanisms purely based on wave breaking, preexisting TKE is amplified and subsequently dissipated by the joint action of a mean Eulerian wind-induced shear current and the Stokes drift of surface waves, the same elements thought to be responsible for the generation of Langmuir circulations. Assuming that the TKE dissipation rate $\varepsilon$ saturates to its equilibrium value over a time of the order one eddy turnover time of the turbulence, a new scaling expression, dependent on the turbulent Langmuir number, is derived for $\varepsilon$. For reasonable values of the input parameters, the new expression predicts an increase of the dissipation rate near the surface by orders of magnitude compared with usual surface-layer scaling estimates, consistent with available OBL data. These results establish on firmer grounds a suspected connection between two central OBL phenomena: dissipation enhancement and Langmuir circulations.
\end{abstract}

\section{Introduction}

A phenomenon that has been reported extensively in the oceanographic literature is the enhancement of the turbulent kinetic energy (TKE) dissipation rate beneath surface waves (Gargett 1989; Agrawal et al. 1992; Melville 1996). This has wide-ranging implications for upperocean mixing and air-sea interaction. Whereas in flat-wall boundary layers the dissipation rate varies with height or depth in the surface layer approximately proportionally to $|z|^{-1}$ (Csanady 2004), much faster variations, proportional to $|z|^{-2}$ or $|z|^{-4}$, have been inferred from ocean turbulence measurements (Gargett 1989; Terray et al. 1996).

Gargett (1989) suggested that the two most probable causes for this dissipation enhancement near the surface are wave breaking and Langmuir circulations, but the second possibility has not been explored in detail. The simplest explanation for the $|z|^{-4}$ power law displayed by the dissipation rate draws an analogy between wave breaking and the stirring caused in the water by an oscillating grid in a water tank (Melville 1996). More elaborate explanations

Corresponding author address: Miguel A. C. Teixeira, CGUL, IDL, University of Lisbon, Edifício C8, Campo Grande, Lisbon, Portugal. E-mail: mateixeira@fc.ul.pt have been developed making use of the equations of motion.

Craig and Banner (1994), for example, analyzed various balances of the terms in the TKE equation and found that, when transport by turbulence produced by wave breaking balances dissipation, a power-law behavior for the dissipation rate profile is obtained, with an exponent of $\approx-3.4$. However, Craig and Banner's model requires the use of rather high values for the roughness length $z_{0}$ in order to fit their data adequately. Although enhancement of $z_{0}$ has been confirmed in direct numerical simulations (DNS) of the oceanic boundary layer (OBL) (Sullivan et al. 2004), alternative theories of TKE dissipation, not so sensitive to $z_{0}$, should not be ruled out. Anis and Moum (1995) proposed two mechanisms for the enhancement of dissipation near the surface. One of them relies on the transport of turbulence produced by breaking waves by the wave motion. The other depends on the existence of a rotational wave field. Both lead to an exponential dissipation rate profile. However, Anis and Moum used two exponentials, with different parameters, to fit their dissipation data in two different depth ranges, and this does not seem very satisfactory.

Terray et al. (1996) used scaling arguments to justify the $|z|^{-2}$ power law found in their dissipation data. The formula obtained by them using dimensional analysis 
was supported to a certain extent also by the measurements of Drennan et al. (1996). The considerable disagreement between different authors concerning the value of the exponent in the power law followed by the dissipation rate suggests that this exponent may not be unique. In other words, the possibility that the dissipation rate may not follow a simple power law deserves to be investigated.

The large-eddy simulation (LES) results of McWilliams et al. (1997), Noh et al. (2004), and Li et al. (2005) and the experiments of Thais and Magnaudet (1996) have shown that, even in the absence of wave breaking, the dissipation rate is still enhanced, although not as much as in the ocean. The fact that in LES studies the waves are not resolved but are parameterized through their vortex force (see Leibovich 1977) is a strong argument in favor of Langmuir circulations as a cause for at least part of this dissipation enhancement.

Here a simple model for explaining the shape and magnitude of the dissipation rate profile is proposed, based on ideas suggested by rapid-distortion theory (RDT) (Teixeira 2011), where turbulence is amplified by the joint action of a mean shear and a Stokes drift gradient with the same sign, leading to very substantial enhancement of the TKE and of its dissipation. Section 2 contains a description of the model. In section 3 , results from the model are tested against three existing datasets. Section 4 contains a summary of the main findings.

\section{A simple model for the dissipation rate}

A widely accepted form for the profile of the viscous dissipation rate $\varepsilon$ in the surface layer of a flat-wall boundary layer is

$$
\varepsilon=\frac{u_{*}^{3}}{\kappa|z|}
$$

(where $u_{*}$ is the friction velocity, $\kappa$ is von Kármán's constant, and $z$ is the height or depth). One way to obtain this equation is by neglecting all the terms other than shear production and dissipation in the TKE equation (see, e.g., Csanady 2004), assuming that the shear stress is constant and equal to $-u_{*}^{2}$ and that the mean velocity profile is logarithmic (both assumptions being consistent with the usual surface-layer scaling).

If, analogously, in the case where both shear and the Stokes drift of surface waves exist, the dissipation rate is balanced by the production terms in the TKE equation (which now include a Stokes drift term; see McWilliams et al. 1997; Teixeira 2011), then

$$
\varepsilon=-\overline{u w}\left(\frac{d U}{d z}+\frac{d U_{S}}{d z}\right),
$$

where $\overline{u w}$ is the turbulent shear stress, $d U / d z$ is the mean shear rate, and $d U_{s} / d z$ is the strain rate associated with the Stokes drift. Formally, this equation is not too different from (1). However, as was shown theoretically by Teixeira (2011) and experimentally by Thais and Magnaudet (1996), $\overline{u w}$ is radically modified by the presence of surface waves, so the differences are greater than they appear at first glance. It should be emphasized that the shear stress used in (2) must be an equilibrium value in turbulence subjected to the strains $d U / d z$ and $d U_{s} / d z$, which are assumed to be aligned in the same direction and to have the same sign (a situation that is known to be linearly unstable to Langmuir circulations). These assumptions approximate the near-surface OBL in situations with surface waves driven by a wind stress (wind waves) (see Teixeira 2011).

Apart from the tendency, the balances expressed by (1) and (2) neglect the turbulent-transport and pressuretransport terms, which LES studies have shown to be a more questionable approximation in the latter case than in the former (Polton and Belcher 2007; Grant and Belcher 2009). However, (2) seems a reasonable first guess, because at least when vertically integrated (excluding boundary fluxes) it must be true; turbulent fluxes and pressure fluxes only move TKE around, they do not generate it. In this respect, what is most important is not the turbulent transport within the water but the flux of TKE into the water. This aspect is taken into account here through the assumption of a prescribed initial shear stress, as will be seen next. Although LES studies suggest that the turbulent-flux terms in the TKE equation are also important locally (influencing the dissipation profiles), there is conflicting evidence. The LES studies of Polton and Belcher (2007) and Grant and Belcher (2009), for example, suggest that there is a region in the OBL whose depth scales on the wavelength of the waves, where the balance in (2) approximately holds, even locally. They term this the Stokes layer. Because wave breaking is a highly intermittent process, turbulent fluxes could be important immediately after the injection of turbulence into the water, but (2) could become valid later, when the TKE has been amplified considerably and dissipation has had to increase as well in order to limit its growth. It is plausible that this later stage could account for a substantial fraction of the averaged dissipation (see Gemmrich and Farmer 2004).

With these cautions, the form of the various terms in (2) is prescribed as follows: First, it is assumed that, even when surface waves are present, the mean velocity in the water flow follows a logarithmic form,

$$
\frac{d U}{d z}=\frac{u_{*}}{\kappa|z|}
$$


This is supported, for example, by Kondo (1976) and Craig and Banner (1994). Although some of the observations collected by Terray et al. (1999) do not support (3), others show that it is valid up to the depths where the dissipation is substantially increased (cf. their Figs. 1 and 3), although not directly beneath the air-water interface. This objection should not be too serious, because the present calculations do not aim at more than a correct scaling behavior.

Second, as a leading-order approximation, the Stokes drift is assumed to take the form appropriate for a monochromatic irrotational surface wave,

$$
\frac{d U_{S}}{d z}=2\left(a_{w} k_{w}\right)^{2} \sigma_{w} e^{-2 k_{w}|z|},
$$

where $a_{w}, k_{w}$, and $\sigma_{w}$ are the amplitude, wavenumber, and angular frequency of the dominant surface waves, respectively. Although a more realistic surface wave spectrum modifies the Stokes drift profile very near the surface (Rascle et al. 2006), at the depths where dissipation rate measurements are performed the waves at the spectral peak are likely to give a fairly dominant contribution.

The definition of the shear stress is a crucial aspect of this model. Because $\overline{u w}$ should be taken at equilibrium and numerous authors (Townsend 1970; Lee et al. 1990; Teixeira and Belcher 2010) have shown that RDT (where the flow is linearized with respect to the turbulence) gives a fair estimate of the equilibrium value of various turbulent statistics as long as these are calculated at a time of the order the eddy turnover time of the turbulence $T_{L}, \overline{u w}$ should be evaluated at that time. It was seen in Teixeira (2011) that, when $(d U / d z)\left(d U_{s} / d z\right)>0$, the shear stress grows approximately exponentially with the distortion by the mean strain $d U / d z+d U_{s} / d z$. The equation that the shear stress satisfies, when nonlinear processes are neglected [Eq. (21) of Teixeira 2011], is

$$
\begin{aligned}
\frac{d^{2} \overline{u w}}{d t^{2}}-4 \frac{d U}{d z} \frac{d U_{S}}{d z} \overline{u w}= & -\frac{1}{\rho} \frac{d}{d t}\left(\overline{w \frac{\partial p}{\partial x}}+\overline{u \frac{\partial p}{\partial z}}\right) \\
& +\frac{2}{\rho}\left(\frac{d U}{d z} \overline{w \frac{\partial p}{\partial z}}+\frac{d U_{S}}{d z} u \overline{\frac{\partial p}{\partial x}}\right),
\end{aligned}
$$

where $p$ is the turbulent pressure and $\rho$ is the density. This equation is consistent with a linearized version of CraikLeibovich's momentum equation, including a vortex force term (see Leibovich 1977). To explain the growth of $\overline{u w}$ and of the TKE with time in his RDT model, Teixeira (2011) additionally neglected the correlation terms involving the pressure [i.e., the right-hand side of (5)]. This is consistent with the idea that the pressure-strain terms are less important than the production terms, especially when these are associated with an instability process, as is the case here. Then (5) becomes approximately

$$
\frac{d^{2} \overline{u w}}{d t^{2}}-4 \frac{d U}{d z} \frac{d U_{S}}{d z} \overline{u w} \approx 0 .
$$

RDT suggests the adoption of the solution to (6), evaluated at a time $T_{L}$, as a definition for $\overline{u w}$ in (2) (cf. Teixeira and Belcher 2010). The exponentially growing solution of (6), which is the physically relevant one, evaluated at equilibrium, may therefore be written as

$$
\overline{u w}=\overline{u w}(t=0) \exp \left[2\left(\frac{d U}{d z} \frac{d U_{s}}{d z}\right)^{1 / 2} T_{L}\right]
$$

Equation (7) implies that the shear stress grows at the same rate as unstable flow disturbances in Langmuir circulations (cf. Leibovich 1977), until it saturates to an equilibrium value.

Finally, the initial shear stress is assumed to be

$$
\overline{u w}(t=0)=-u_{*}^{2}
$$

(independent of depth). The time evolution of the shear stress is therefore approximated as that of a shear-driven boundary layer over which a Stokes drift is suddenly imposed (this concept is consistent with both RDT and the experiments of Melville et al. 1998).

Inherent to the RDT approach is the idea that the evolution of the shear stress is determined by the initial forms of the mean velocity and Stokes drift profiles, even if in reality the former may be modified by vertical mixing during the course of the distortion that yields the final equilibrium state, and the latter may weaken because of energy transfer to the turbulence (Teixeira and Belcher 2002).

As will be seen, the choices (3) and (8) have the advantage of making the dissipation rate tend to the usual surface-layer form when $z \rightarrow-\infty$. This feature is supported by measurements (Agrawal et al. 1992).

Inserting (7) into (2) and using (3), (4), and (8) yields

$$
\begin{aligned}
\varepsilon= & u_{*}^{2}\left[\frac{u_{*}}{\kappa|z|}+2\left(a_{w} k_{w}\right)^{2} \sigma_{w} e^{-2 k_{w}|z|}\right] \\
& \times \exp \left[2\left(\frac{2 u_{*} \sigma_{w}}{\kappa|z|}\right)^{1 / 2} a_{w} k_{w} e^{-k_{w}|z|} T_{L}\right] .
\end{aligned}
$$

The dissipation rate in the OBL is often presented in dimensionless form, normalized by $u_{*}$ and $z$, as a function of $z$ normalized by $u_{*}$ and $g$ (the acceleration of gravity) (Agrawal et al. 1992; Melville 1996). In terms of these dimensionless variables, (9) takes the form 


$$
\frac{\varepsilon \kappa|z|}{u_{*}^{3}}=\left[1+2\left(a_{w} k_{w}\right)^{2}\left(\frac{u_{*}}{c_{w}}\right) \kappa\left(\frac{g|z|}{u_{*}^{2}}\right) e^{-2\left(u_{*} / c_{w}\right)^{2}\left(g|z| \mid u_{*}^{2}\right)}\right] \exp \left\{2\left[\frac{2}{\kappa}\left(\frac{c_{w}}{u_{*}}\right)^{3}\left(\frac{u_{*}^{2}}{g|z|}\right)\right]^{1 / 2} a_{w} k_{w} e^{-\left(u_{*} / c_{w}\right)^{2}\left(g|z| / u_{*}^{2}\right)} u_{*} k_{w} T_{L}\right\},
$$

where the linear dispersion relation of deep-water surface gravity waves, $\sigma_{w}^{2}=g k_{w}$, has been used and $c_{w}=\sigma_{w} / k_{w}$ is the phase speed of the waves. It can be seen that the dimensionless dissipation rate depends on three dimensionless parameters, which must be prescribed, $a_{w} k_{w}$, $u_{*} / c_{w}$, and $u_{*} k_{w} T_{L}$. Even if depth and the dissipation rate had been normalized using the significant wave height, as in Terray et al. (1996), related parameters would appear. This suggests that scalings for the dissipation where this quantity aims to be treated just as a function of normalized depth are probably an oversimplification. According to (10), the sea state (through the wave slope), the wave age (through $u_{*} / c_{w}$ ), and even the turbulence characteristics in the OBL (through the dimensionless eddy turnover time $\left.u_{*} k_{w} T_{L}\right)$ are essential to properly scale the dissipation rate, as was hinted, for example, by Drennan et al. (1996).

From (9) and the definition of the turbulent Langmuir number $\mathrm{La}_{t}=\left[u_{*} / U_{S}(z=0)\right]^{1 / 2}=\left\{u_{*} /\left[\left(a_{w} k_{w}\right)^{2} c_{w}\right]\right\}^{1 / 2}$, an alternative expression for the dissipation rate may be derived, which emphasizes the connection with Langmuir turbulence (McWilliams et al. 1997) and reduces the number of input parameters to two,

$$
\begin{aligned}
\frac{\varepsilon \kappa|z|}{u_{*}^{3}}= & {\left[1+2 \kappa \operatorname{La}_{t}^{-2}\left(k_{w}|z|\right) e^{-2 k_{w}|z|}\right] } \\
& \times \exp \left\{2 \mathrm{La}_{t}^{-1}\left[\frac{2}{\kappa\left(k_{w}|z|\right)}\right]^{1 / 2} e^{-k_{w}|z|} u_{*} k_{w} T_{L}\right\} .
\end{aligned}
$$

In this formulation, it can be seen that the relevant scaling for depth is $k_{w}|z|$, and the only dimensionless parameter in addition to $\mathrm{La}_{t}$ is $u_{*} k_{w} T_{L}$. The parameter $\mathrm{La}_{t}$ therefore encapsulates effects both of the wave slope and of the wave age. When $\mathrm{La}_{t} \rightarrow \infty$, (11) tends to the usual surfacelayer scaling (1), whereas, if $\mathrm{La}_{t}$ is small, the dissipation is strongly enhanced near the surface.

\section{Comparison with field data and discussion}

Next, results from this simple model are compared with field data collected by Agrawal et al. (1992), Terray et al. (1996), and Drennan et al. (1996). Figure 1a shows a comparison between the data of Agrawal et al. (1992), as reproduced in Fig. 7 of Craig and Banner (1994) (symbols), and the theoretical prediction given by (10). Also shown in Fig. 1a are predictions from the model of
Craig and Banner (1994) for two values of $z_{0}$ (dashed lines), extracted from their Fig. 7, and the prediction from the usual surface-layer scaling (dotted line).

The input parameters of (10) were estimated in the following way: Agrawal et al. (1992) mention that the waves to which their measurements refer have a significant wave height of about $H_{s}=0.3 \mathrm{~m}$. The significant wave height is related to the standard deviation of the surface elevation $\zeta$ by $H_{s}=4\left(\overline{\zeta^{2}}\right)^{1 / 2}$ (Csanady 2004). On the other hand, for a sinusoidal wave, such as assumed in the present model, $\left(\overline{\zeta^{2}}\right)^{1 / 2}=(1 / \sqrt{2}) a_{w}$; therefore, $a_{w}=[1 /(2 \sqrt{2})] H_{s}$ or, for the present case, $a_{w}=0.106 \mathrm{~m}$. From Fig. 1 of Agrawal et al. (1992), it can also be seen that the peak of the wave frequency spectrum is at about $f_{w}=0.55 \mathrm{~Hz}$. This gives $\sigma_{w}=2 \pi f_{w}=3.46 \mathrm{rad} \mathrm{s}^{-1}$, or $k_{w}=1.22 \mathrm{~m}^{-1}$ using the linear dispersion relation of gravity waves. So $a_{w} k_{w}=0.13$ is the value adopted in (10) for Fig. 1a. From the obtained values of $\sigma_{w}$ or $k_{w}$, it can be deduced that $c_{w}=2.84 \mathrm{~m} \mathrm{~s}^{-1}$. Then, because $u_{*}=0.013 \mathrm{~m} \mathrm{~s}^{-1}$ is a fairly typical value of the friction velocity in the water, $u_{*} / c_{w}=$ 0.0046 is estimated here and adopted in (10).

In this, as in the other comparisons presented in this paper, the dimensionless eddy turnover time is treated as an adjustable parameter and set to $u_{*} k_{w} T_{L}=0.24$. The eddy turnover time $T_{L}$ should scale as $l / u_{*}$, where $l$ is a representative length scale of the turbulence in the water. The above estimate implies that $k_{w} l=0.24$, or $l=0.038 \lambda_{w}$, where $\lambda_{w}$ is the wavelength of the dominant surface waves. Perhaps a fixed relationship between $l$ and $\lambda_{w}$, with $l$ being a relatively small fraction of $\lambda_{w}$, is expectable in the present context, given that the source of the turbulence whose TKE is amplified and dissipated appears to be chiefly wave breaking (Agrawal et al. 1992; Craig and Banner 1994), which is a rather localized process. Gargett and Wells (2007) suggest instead that, in coastal waters, $l \approx \lambda_{w}$ when Langmuir circulations exist, but, because in the present model both $l$ and $u_{*}$ pertain to an initial wave-undistorted state, the above estimate for $l$ should be more appropriate.

With these values chosen for the parameters, (10) predicts that the dissipation rate is enhanced by several orders of magnitude near the surface. This enhancement is clearly mainly due, through (7), to a corresponding increase of the shear stress in the same region.

Part of the large scatter displayed by the data in Fig. 1a should be attributed to the different values of $a_{w} k_{w}$ and 

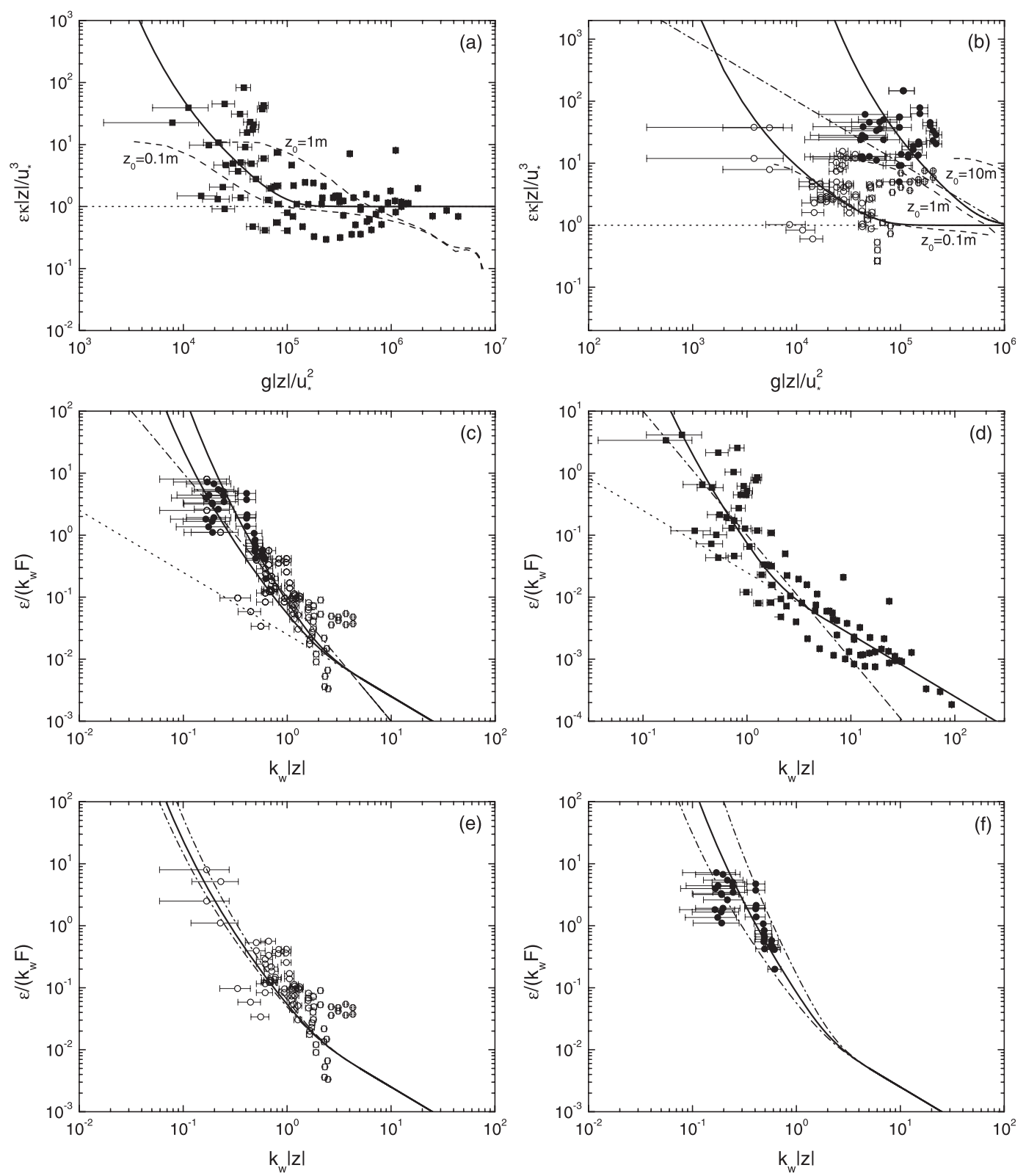

FIG. 1. Normalized dissipation rate as a function of normalized depth for the present model (solid lines), the usual surface-layer scaling (dotted lines), the model of Craig and Banner (1994) (dashed lines), the data from Agrawal et al. (1992) (squares), the data from Terray et al. (1996) (open circles), and the data from Drennan et al. (1996) (filled circles). Error bars correspond to $z \pm a_{w}$. Shown are (a),(b) $\varepsilon \kappa|z| / u_{*}^{3}$ vs $g|z| / u_{*}^{2}$, where the dashed-dotted line is the open-ocean relation from Burgers (1997); (c),(d) $\varepsilon /\left(k_{w} F\right)$ vs $k_{w}|z|$, where the dashed-dotted line is the power-law relation by Drennan et al. (1996) (see text); and (e),(f) $\varepsilon /\left(k_{w} F\right)$ vs $k_{w}|z|$ from WAVES and SWADE, respectively, where the dashed-dotted lines are the present model, using lower and upper bounds of $\mathrm{La}_{t}$ for each dataset.

$u_{*} / c_{w}$, (and probably of $u_{*} k_{w} T_{L}$ ) associated with the data, but these values were not provided in the study of Agrawal et al. (1992). Obviously, different values of these parameters would produce different theoretical predictions according to (10). However, the plausible range of variation of these parameters is too large to get a useful idea of the corresponding spread of those predictions. For that reason, it was decided to include a single representative curve from (10) in Fig. 1a. Another part of the scatter, specifically that along the horizontal axis, should be related to the fact that the data of Agrawal et al. (1992) were measured at fixed depths, whereas (10) implicitly assumes a surface-following coordinate system (see Teixeira and Belcher 2010; Teixeira 2011). An estimate of this part 
of the uncertainty is provided in Fig. 1 by the error bars associated with the data, which correspond to the addition or subtraction of $a_{w}$ from the depth $z$. The error bars are generally small in relative terms, except at the lowest measurement depths.

In Fig. 1b, the dissipation data from the Water Air Vertical Exchange Studies (WAVES) (Terray et al. 1996) and Surface Waves Dynamics Experiment (SWADE) (Drennan et al. 1996) field campaigns, extracted from Fig. 4 of Drennan et al. (1996) (symbols), are compared with predictions from (10) (solid lines). Results from the model of Craig and Banner (1994) (also extracted from Fig. 4 of Drennan et al. 1996) (dashed lines), the openocean relationship of Burgers (1997) (dashed-dotted line), and the usual surface-layer scaling (dotted line) are also shown.

The values of $a_{w} k_{w}$ and $u_{*} / c_{w}$ were calculated objectively from the WAVES and SWADE datasets [Table 1 of Terray et al. (1996) and Table 1 of Drennan et al. (1996)] as the arithmetic mean of each quantity for all runs, after identifying the wavenumber and phase speed at the spectral peak with $k_{w}$ and $c_{w}$, respectively, and relating $a_{w}$ to $H_{s}$ in the way described above. This yields $a_{w} k_{w}=0.109$ and $u_{*} / c_{w}=0.00573$ for the WAVES dataset and $a_{w} k_{w}=0.090$ and $u_{*} / c_{w}=0.00184$ for the SWADE dataset, values that were used in (10) for Fig. 1b, along with $u_{*} k_{w} T_{L}=0.24$. The error bars for the depth were calculated in the same way as for Fig. 1a, except that the arithmetic mean value of the required flow parameters from each dataset was employed.

Again, there is large scatter in the data, which may be attributed partly to the different values of $a_{w} k_{w}, u_{*} / c_{w}$, and $u_{\circledast} k_{w} T_{L}$ for the various data points and partly to the fact that the measurements were taken at fixed depths. The large observed disparity between the two datasets appears to result from the very different values of $u_{*} / c_{w}$ existing in WAVES and SWADE (as will be seen in more detail next). The definition of the dimensionless vertical coordinate as $g|z| / u_{*}^{2}$ gives a somewhat artificial sensitivity of the dissipation rate profiles to $u_{*} / c_{w}$. However, other reasons for this disparity, for example associated with the variation of $u_{*} k_{w} T_{L}$, cannot be excluded.

As noted by Terray et al. (1996) and Drennan et al. (1996), this problem can be attenuated if the scaling for $z$ is changed. Figure 1c reproduces Fig. 6 of Drennan et al. (1996), where $\varepsilon /\left(k_{w} F\right)$ ( $F$ being the energy flux into the waves) is plotted as a function of $k_{w}|z|$. In this figure, the usual surface-layer scaling (dotted line) and the power-law fit suggested by Drennan et al. (1996), $\varepsilon /\left(k_{w} F\right)=0.1\left(k_{w}|z|\right)^{-2}$ (dashed-dotted line), are shown. The results from (10) (solid lines) were obtained noting that $g|z| / u_{*}^{2}=\left(c_{w} / u_{*}\right)^{2}\left(k_{w}|z|\right)$ and that, if $F=\alpha u_{*}^{3}$ with $\alpha=100$ (Drennan et al. 1996), then $\varepsilon /\left(k_{w} F\right)=\left(\varepsilon \kappa|z| / u_{*}^{3}\right) /\left(\alpha \kappa k_{w}|z|\right)$. The error bars were calculated in the same way as for Fig. 1b but have a different size here because in the conversion between Figs. 4 and 6 of Drennan et al. (1996) the variation of $a_{w}, u_{*}$, and $k_{w}$ within the WAVES and SWADE datasets was taken into account, whereas average values for each dataset were employed in the present calculation.

Figure $1 \mathrm{c}$ shows that use of the vertical coordinate $k_{w}|z|$ [the most natural one in the present model; see (11)] reduces the data scatter considerably, as well as the differences between the two predictions from (10) (using the same values of $a_{w} k_{w}, u_{*} / c_{w}$, and $u_{*} k_{w} T_{L}$ as in Fig. 1b). For this particular case, the predictions of the present model, although definitely with a clearer physical basis, are of comparable accuracy to that of the expression of Drennan et al. (1996).

In Fig. 1d, the data of Agrawal et al. (1992) are replotted using the variables $k_{w}|z|$ and $\varepsilon /\left(k_{w} F\right)$, along with the analytical expression of Drennan et al. (1996). Despite the fact that the conversion from Fig. 1a to Fig. 1d used a single value of $u_{*} / c_{w}=0.0046$ (estimated before), the apparent scatter of the data is reduced in this representation. It can be noticed that (10) captures more accurately the transition between the usual surface-layer behavior of the dissipation rate and its enhancement near the surface.

It was pointed out in section 2 that the turbulent Langmuir number $\mathrm{La}_{t}$ is a key parameter in the scaling for the dissipation proposed here. For the conditions considered in the present model in Figs. 1a,d, for example, $\mathrm{La}_{t}=\left(u_{*} / c_{w}\right)^{1 / 2} /\left(a_{w} k_{w}\right)=0.52$, whereas in Figs. 1b,c $\mathrm{La}_{t}=0.69$ for the WAVES dataset and $\mathrm{La}_{t}=0.48$ for the SWADE dataset, which seem reasonable values (McWilliams et al. 1997; Li et al. 2005). It is noteworthy that, according to the regime diagram of Li et al. (2005) (their Fig. 5), these Langmuir numbers correspond to turbulence that is more isotropic than shear-driven turbulence, but its structure is different from that of proper Langmuir turbulence, as defined by McWilliams et al. (1997), for which $\mathrm{La}_{t} \approx 0.3$.

In fact, from the WAVES and SWADE datasets it is possible to calculate the range of variation of $\mathrm{La}_{t}$. This allows getting an idea of how much of the data scatter in Fig. 1c may be due to this aspect. Figure 1e shows again data from the WAVES dataset (symbols), along with the prediction of (11) presented in Fig. 1c (solid line) and predictions corresponding to lower and upper bounds for $\mathrm{La}_{t}$ in the dataset ( 0.59 and 0.77 , respectively; dashed-dotted lines). In Fig. 1f, the same is shown for the SWADE dataset (where the bounds of $\mathrm{La}_{t}$ are 0.33 and 0.66 instead). It is clear that, although a large fraction of the scatter might be attributed to the variation of $\mathrm{La}_{t}$ in SWADE (especially if the error bars are taken into account), the same cannot be said about WAVES. 
Obviously, many other possible sources of scatter exist, as was pointed out above.

Although the preceding comparisons with data suggest that shear-Stokes drift-turbulence interaction may be sufficient to explain the order of magnitude of the dissipation rate, the concurrent action of other mechanisms is obviously not ruled out and could be easily accommodated through a suitable recalibration of $u_{*} k_{w} T_{L}$.

\section{Conclusions}

A new mechanism for explaining the observed enhancement of the TKE dissipation rate in the OBL was proposed, and a corresponding scaling expression was developed, based on RDT arguments. In the simple model developed here, dissipation is assumed to be balanced, at equilibrium, by shear and by Stokes drift TKE production. The strong amplification of the shear stress that is predicted by RDT when the mean shear and Stokes drift gradient have the same sign (Teixeira 2011) is responsible for a large increase of the dissipation rate near the surface. A substantial part of the dissipation enhancement occurring in the OBL could thus result from this shear-Stokes drift-turbulence interaction, the same mechanism that leads to the development Langmuir turbulence: that is, Langmuir circulations on a wide range of scales (cf. Gargett 1989). The present study is a contribution to the establishment of a more definite link between these two central phenomena that characterize the OBL. The proposed dissipation enhancement mechanism acts in conjunction with wave breaking, which is one of the likeliest sources for the TKE that is amplified by the mechanism, before being dissipated. Although the comparisons with data presented above suggest that this mechanism is sufficient to explain the order of magnitude of the dissipation rate, the concurrent action of other mechanisms is obviously not ruled out.

For values of the input parameters determined objectively from the adopted datasets, the dissipation rate scaling developed here succeeds in broadly reproducing the shape and order of magnitude of the dissipation rate profiles measured by Agrawal et al. (1992), Terray et al. (1996), and Drennan et al. (1996), which nevertheless have a large scatter. Despite not being very recent, WAVES and SWADE are among the most complete existing datasets relevant for TKE dissipation in the OBL, including information, not only on turbulence parameters of the ocean surface layer but also on the wave parameters characterizing the accompanying sea state. According to the scaling developed here, the normalized dissipation rate depends not only on a normalized depth $k_{w}|z|$ but also on the wave slope and wave age (as suggested by Drennan et al. 1996) or alternatively on the turbulent
Langmuir number $\mathrm{La}_{t}$, as well as on a dimensionless eddy turnover time of the turbulence. Hence, the model suggests that, in order to correctly scale the dissipation rate, all of these input parameters should be taken into account. Their variation in the examined datasets probably explains part of the scatter that remains in the scaled data. It seems likely that the dissipation enhancement mechanism proposed here is continuously active in the ocean (although of variable strength), because of the ubiquity of wind-induced shear currents and propagating surface waves.

Acknowledgments. I acknowledge the financial support of the Portuguese Science Foundation (FCT) under Project PTDC/EME-MFE/099636/2008.

\section{REFERENCES}

Agrawal, Y. C., E. A. Terray, M. A. Donelan, P. A. Hwang, A. J. Williams III, W. M. Drennan, K. K. Kahma, and S. A. Kitaigorodskii, 1992: Enhanced dissipation of kinetic energy beneath surface waves. Nature, 359, 219-220.

Anis, A., and J. N. Moum, 1995: Surface wave-turbulence interactions: Scaling $\varepsilon(z)$ near the sea surface. J. Phys. Oceanogr., 25, 2025-2045.

Burgers, G., 1997: Comments on "Estimates of kinetic energy dissipation under breaking waves." J. Phys. Oceanogr., 27, 2306-2307.

Craig, P. D., and N. L. Banner, 1994: Modeling wave-enhanced turbulence in the ocean surface layer. J. Phys. Oceanogr., 24, 2546-2559.

Csanady, G. T., 2004: Air-Sea Interaction: Laws and Mechanisms. Cambridge University Press, 239 pp.

Drennan, W. M., M. A. Donelan, E. A. Terray, and K. B. Katsaros, 1996: Oceanic turbulence dissipation measurements in SWADE. J. Phys. Oceanogr., 26, 808-815.

Gargett, A. E., 1989: Ocean turbulence. Annu. Rev. Fluid Mech., 21, 419-451.

_ Part 1. Observations. J. Fluid Mech., 576, 27-61.

Gemmrich, J. R., and D. M. Farmer, 2004: Turbulence in the presence of breaking waves. J. Phys. Oceanogr., 34, 10671086.

Grant, A. L. M., and S. E. Belcher, 2009: Characteristics of Langmuir turbulence in the ocean mixed layer. J. Phys. Oceanogr., 39, 1871-1887.

Kondo, J., 1976: Parameterization of turbulent transport in the top meter of the ocean. J. Phys. Oceanogr., 6, 712-720.

Lee, M. J., J. Kim, and P. Moin, 1990: Structure of turbulence at high shear rate. J. Fluid Mech., 216, 561-583.

Leibovich, S., 1977: Convective instability of stably stratified water in the ocean. J. Fluid Mech., 82, 561-581.

Li, M., C. Garrett, and E. Skyllingstad, 2005: A regime diagram for classifying turbulent large eddies in the upper ocean. Deep-Sea Res., 52, 259-278.

McWilliams, J. C., P. P. Sullivan, and C.-H. Moeng, 1997: Langmuir turbulence in the ocean. J. Fluid Mech., 334, 1-30.

Melville, W. K., 1996: The role of surface-wave breaking in air-sea interaction. Annu. Rev. Fluid Mech., 28, 279-321. 
_, R. Shear, and F. Veron, 1998: Laboratory measurements of the generation and evolution of Langmuir circulations. J. Fluid Mech., 364, 31-58.

Noh, Y., H. S. Min, and S. Raasch, 2004: Large eddy simulation of the ocean mixed layer: The effects of wave breaking and Langmuir circulation. J. Phys. Oceanogr., 34, 720-735.

Polton, J. A., and S. E. Belcher, 2007: Langmuir turbulence and deeply penetrating jets in an unstratified mixed layer. J. Geophys. Res., 112, C09020, doi:10.1029/2007JC004205.

Rascle, N., F. Ardhuin, and E. A. Terray, 2006: Drift and mixing under the ocean surface: A coherent one-dimensional description with application to unstratified conditions. J. Geophys. Res., 111, C03016, doi:10.1029/2005JC003004.

Sullivan, P. P., J. C. McWilliams, and W. K. Melville, 2004: The oceanic boundary layer driven by wave breaking with stochastic variability. Part 1. Direct numerical simulations. J. Fluid Mech., 507, 143-174.

Teixeira, M. A. C., 2011: A linear model for the structure of turbulence beneath surface water waves. Ocean Modell., 36, 149-162.
— a progressive surface wave. J. Fluid Mech., 458, 229-267.

- , and - 2010: On the structure of Langmuir turbulence. Ocean Modell., 31, 105-119.

Terray, E. A., M. A. Donelan, Y. C. Agrawal, W. M. Drennan, K. K. Kahma, A. J. Williams III, P. A. Hwang, and S. A. Kitaigorodskii, 1996: Estimates of kinetic energy dissipation under breaking waves. J. Phys. Oceanogr., 26, 792-807.

— W. M. Drennan, and M. A. Donelan, 1999: The vertical structure of shear and dissipation in the ocean surface layer. Proc. Symp. on the Wind-Driven Air-Sea Interface-Electromagnetic and Acoustic Sensing, Wave Dynamics and Turbulent Fluxes, Sydney, Australia, University of New South Wales, 239245 .

Thais, L., and J. Magnaudet, 1996: Turbulent structure beneath surface gravity waves sheared by the wind. J. Fluid Mech., 328, 313-344.

Townsend, A. A., 1970: Entrainment and the structure of turbulent flow. J. Fluid Mech., 41, 13-46. 